\title{
Ruolo delle tecniche di sequenziamento di ultima generazione nella gestione del paziente con carcinoma tiroideo
}

\author{
Raffaele Ciampi ${ }^{1} \cdot$ Rossella Elisei $^{1}$
}

Accettato: 2 febbraio 2021 / Pubblicato online: 27 maggio 2021

(c) The Author(s) 2021

La genetica del carcinoma tiroideo è stata a lungo oggetto di studio e molti dei meccanismi patogenetici sono conosciuti da tempo ma negli ultimi anni, in virtù dell'avanzamento tecnologico avvenuto nel campo della biologia molecolare, lo studio dei meccanismi patogenetici di questa malattia ha avuto un notevole impulso.

Una di queste rivoluzioni tecnologiche ha portato alla tecnologia denominata next generation sequencing (NGS) che, nell'ultimo decennio, ha rappresentato un vero e proprio spartiacque nella ricerca oncologica e non solo. L'NGS permette il sequenziamento simultaneo di centinaia di geni partendo da quantità minime di campione; grazie a questa tecnologia è possibile sequenziare l'intero genoma (wholegenome sequencing, WGS), la sua porzione codificante (whole-exome sequencing, WES) oppure porzioni discrete del genoma che includono specifici geni di interesse o addirittura di porzioni di geni specificamente alterati in una data neoplasia (target sequencing), Tabella 1. Queste sono le applicazioni principali ma con questa tecnologia è possibile sequenziare anche l'RNA (trascrittoma), valutare modificazioni epigenetiche (epigenoma), il corredo dei miRNA (miRNoma) o l'interazione tra le proteine (proteoma).

Le tecnologie NGS sono state utilizzate in maniera sistematica in questi anni dal progetto del Cancer Genome Atlas Network che negli USA ha definito il genetic landscape di più di 33 tipi di carcinoma umano portando a un enorme avanzamento delle conoscenze alla base della fisiopatologia dell'iniziazione e progressione tumorale. Anche il genetic landscape di circa 400 PTC è stato definito in uno studio importantissimo, in cui l'analisi con un approccio integrato

\footnotetext{
$凶$ R. Ciampi

raffaele.ciampi@unipi.it

1 Unità di Endocrinologia, Dipartimento di Medicina Clinica e Sperimentale, Università di Pisa, Pisa, Italia
}

di "omica" utilizzando diverse piattaforme NGS ha fornito alla comunità scientifica una fotografia dettagliatissima dei meccanismi genetici alla base della carcinogenesi di questo tipo di tumore [1]. Il ruolo di geni driver come $B R A F, R A S$, $R E T$ e $N T R K 1 / 3$ è stato confermato e nuovi geni driver come EIF1AX, PPM1D e CHECK2 sono stati scoperti. Grazie alla maggiore sensibilità rispetto alle tecniche di sequenziamento diretto, il numero di casi negativi (dark matter) si è ridotto moltissimo passando dal 25 al 3,5\%. Inoltre, grazie all'integrazione dei dati di genomica, trascrittomica, proteomica, ecc., gli autori hanno proposto una nuova riclassificazione del PTC in due grandi cluster in base ai loro driver genetici (BRAFV600E-like e RAS-like) che sono responsabili di diversi livelli di attivazione della MAPK pathway.

L'accesso sempre maggiore a piattaforme NGS ha consentito la genotipizzazione su larga scala di campioni di archivio e molti studi hanno aggiornato i dati precedentemente ottenuti sulla patogenesi tumorale. L'approccio più utilizzato è quello del target sequencing che risulta meno costoso e più semplice da adattare alle esigenze della pratica clinica, anche in virtù della maggior semplicità richiesta nell' analisi dei dati. Gli studi più importanti nel carcinoma tiroideo sono stati condotti su PDTC e ATC ma anche sui carcinomi oncocitici a cellule di Hurthle e su carcinomi pediatrici. Studi di NGS, sia WGS o target sequencing, sono stati impiegati anche nella ricerca di mutazioni alternative ai driver consolidati del carcinoma midollare sporadico della tiroide (MTC), quali RET e RAS e, sebbene in alcuni casi siano state trovate alcune alterazioni genetiche alternative, questi studi hanno soprattutto confermato il ruolo centrale dei driver classici. Nel nostro centro abbiamo analizzato 181 MTC sporadici con un approccio di target sequencing grazie a un pannello multi-genico [2]: anche in questo caso, l'utilizzo dell'NGS ha considerevolmente ridotto il numero di casi di MTC che risultavano negativi alla tecnica classica di 
Tabella 1 Principali applicazioni NGS. $S N V$, single nucleotide variants. $C N V$, copy number variations

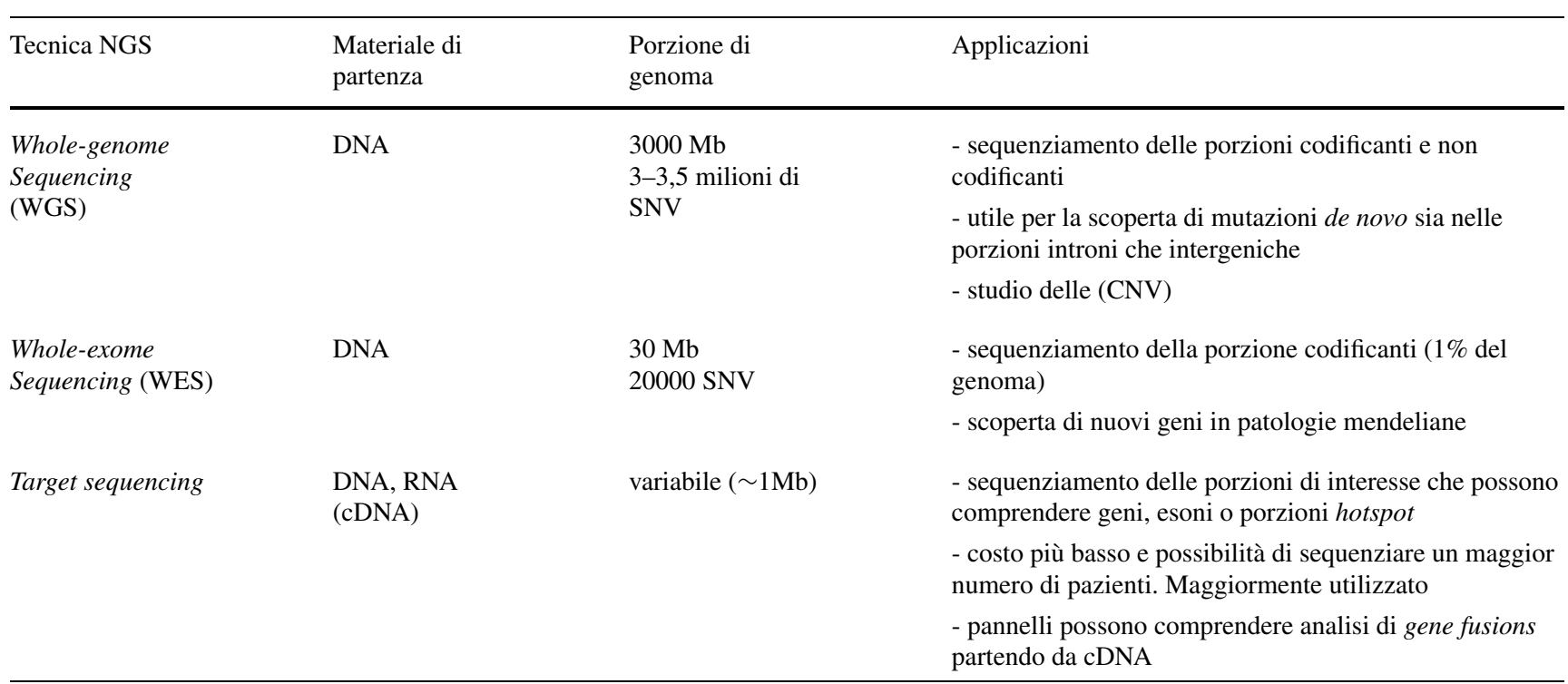

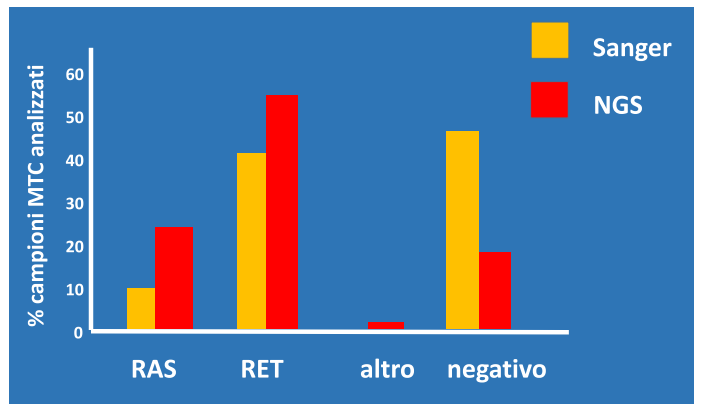

Fig. 1 Confronto tra sequenziamento diretto secondo Sanger e NGS nella ricerca di mutazioni nel carcinoma midollare della tiroide sporadico. Dati elaborati da [2]

sequenziamento (sequenziamento secondo Sanger) (Fig. 1). In aggiunta l'NGS, permettendo anche la valutazione della frequenza allelica delle mutazioni, ci ha permesso di osservare che la frequenza allelica è più alta in tumori più grandi e con un outcome peggiore rappresentando, di per sè, un fattore prognostico negativo [2].

Per quanto riguarda le applicazioni cliniche nel carcinoma tiroideo, l'NGS è ormai raccomandato sia da linee guida nazionali che internazionali $[3,4]$ nella caratterizzazione pre-chirurgica dei noduli tiroidei per evitare chirurgie non necessarie in quel $25 \%$ di casi in cui la citologia su ago aspirato (FNA) rimane indeterminata. Test di diagnostica molecolare (genome classifier) in NGS, come Thyroseq v.3 e Afirma, si basano sulla caratterizzazione del profilo mutazionale o di espressione genica tramite l'utilizzo di pannelli multi-genici e sono sempre più performanti e precisi [5]. La limitazione al loro utilizzo, soprattutto in Europa, rimane principalmente il costo e la necessità di inviare i campioni negli USA. Per ovviare, sempre più centri adottano nella pratica clinica questo tipo di approccio ma utilizzando strategie homemade [4].

L'NGS trova applicazione anche nella gestione della terapia; negli ultimi anni il carcinoma tiroideo, insieme ad altri tipi di tumore, fa sempre più affidamento sulle targeted therapies che si basano sull'identificazione di mutazioni presenti all'interno del tumore da utilizzare come bersaglio per il trattamento con farmaci inibitori.

Inibitori multichinasici come vandetanib e cabozantinib, insieme ai nuovi inibitori selettivi di $B R A F, R E T$ o per i riarrangiamenti di $N T R K$ rappresentano solo alcune delle opzioni terapeutiche a disposizione del clinico per il trattamento del carcinoma tiroideo e questa lista di composti è in continuo aggiornamento. L'accesso a queste terapie dipende strettamente da un preventivo screening genetico del tumore che serve per la selezione del giusto farmaco. Molti pannelli multi-genici sono adesso commercialmente disponibili e/o personalizzabili; si va dai pannelli tumore-specifici a pannelli pancancer che coprono i principali hotspot coinvolti nella carcinogenesi umana nel suo insieme.

Sfortunatamente, una delle criticità legate all'utilizzo di questi farmaci nella pratica clinica è legata a fenomeni di resistenza causati da mutazioni secondarie che via via emergono proprio a causa della pressione selettiva dettata dall'uso del farmaco. L'alta sensibilità dell'NGS permette di valutare la presenza di DNA tumorale circolante (ctDNA) e/o di cellule tumorali circolanti (CTC) contenute nel plasma dei pazienti e ottenibili mediante biopsie liquide, ovvero un semplice prelievo di sangue. Dal ctDNA si può valutare la mutazione driver del tumore primitivo, così come di altre lesioni metastatiche; si possono anche identificare mutazioni secondarie sviluppate nel corso della terapia e che possono essere causa della resistenza farmacologica. Un altro aspet- 
to importante di questa tecnica è il basso tasso di invasività rispetto alle procedure bioptiche standard. La biopsia liquida è già entrata nella pratica clinica nel tumore del polmone non a piccole cellule (NSCLC) e della mammella ma la loro applicazione può essere utile anche in altri tipi di tumore compresi quelli tiroidei [6]. Pochi dati sono ancora presenti in letteratura sull'applicazione delle biopsie liquide nel carcinoma tiroideo e la loro applicazione clinica è ancora dibattuta per una serie di motivi legati ai costi, alla difficoltà tecnica e al fatto che il rilascio di ctDNA nel plasma dipende dal tipo di tumore e, quindi, è variabile caso per caso. Questa tecnica consente di valutare la progressione tumorale tramite l'innalzamento dei valori di frequenza allelica dei marker molecolari e può essere utile anche nello scoprire eventuali mutazioni secondarie di resistenza e indirizzare il paziente verso nuovi farmaci prima che sopraggiunga la progressione della malattia.

In conclusione, l'applicazione delle tecniche NGS non solo ha portato a un miglioramento delle nostre conoscenze sui meccanismi molecolari alla base della patogenesi del carcinoma tiroideo ma è divenuta un elemento fondamentale anche nella pratica clinica, sia per la diagnostica che per la terapia del carcinoma tiroideo, in particolare di quello avanzato.

Funding Note Open access funding provided by Università di Pisa within the CRUI-CARE Agreement.

Conflitto di interesse Gli autori Raffaele Ciampi e Rossella Elisei dichiarano di non avere conflitti di interesse.

Consenso informato Lo studio presentato in questo articolo non ha richiesto sperimentazione umana.

Studi sugli animali Gli autori di questo studio non hanno eseguito studi sugli animali.
Nota della casa editrice Springer Nature rimane neutrale in riguardo alle rivendicazioni giurisdizionali nelle mappe pubblicate e nelle affiliazioni istituzionali.

Open Access This article is licensed under a Creative Commons Attribution 4.0 International License, which permits use, sharing, adaptation, distribution and reproduction in any medium or format, as long as you give appropriate credit to the original author(s) and the source, provide a link to the Creative Commons licence, and indicate if changes were made. The images or other third party material in this article are included in the article's Creative Commons licence, unless indicated otherwise in a credit line to the material. If material is not included in the article's Creative Commons licence and your intended use is not permitted by statutory regulation or exceeds the permitted use, you will need to obtain permission directly from the copyright holder. To view a copy of this licence, visit http://creativecommons.org/licenses/by/4.0/.

\section{Bibliografia}

1. Cancer Genome Atlas Research N (2014) Integrated genomic characterization of papillary thyroid carcinoma. Cell 159:676-690

2. Ciampi R, Romei C, Ramone T et al (2019) Genetic landscape of somatic mutations in a large cohort of sporadic medullary thyroid carcinomas studied by next-generation targeted sequencing. iScience. https://doi.org/10.1016/j.isci.2019.09.030

3. Paschke R, Cantara S, Crescenzi A et al (2017) European Thyroid Association Guidelines regarding thyroid nodule molecular fine-needle aspiration cytology diagnostics. Eur Thyroid $\mathbf{J}$ 6:115-129

4. Pacini F, Basolo F, Bellantone R et al (2018) Italian consensus on diagnosis and treatment of differentiated thyroid cancer: joint statements of six Italian societies. J Endocrinol Invest 41:849-876

5. Muzza M, Colombo C, Pogliaghi G et al (2020) Molecular markers for the classification of cytologically indeterminate thyroid nodules. J Endocrinol Invest 43:703-716

6. Mosele F, Remon J, Mateo J et al (2020) Recommendations for the use of next-generation sequencing (NGS) for patients with metastatic cancers: a report from the ESMO Precision Medicine Working Group. Ann Oncol Off J Eur Soc Med Oncol 31:1491-1505 